\title{
Age patterns in overweight and wasting prevalence of under 5- year-old children from low- and middle-income countries
}

\author{
Luiza I. C. Ricardo $\mathbb{( D D}^{1 凶}$, Giovanna Gatica-Domínguez ${ }^{1}{ }^{1}$, Inácio Crochemore-Silva $\left(\mathbb{D}^{2}{ }^{2}\right.$, Paulo A. R. Neves ${ }^{1}$, Juliana dos Santos Vaz $\left(\mathbb{D}^{1}{ }^{1}\right.$,
} Aluisio J. D. Barros ${ }^{1}$ and Cesar Gomes Victora (iD ${ }^{1}$

(c) The Author(s) 2021

OBJECTIVES: To describe how overweight and wasting prevalence varies with age among children under 5 years in low- and middle-income countries (LMICs).

METHODS: We used data from nationally representative Demographic and Health Surveys and Multiple Indicator Cluster Surveys. Overweight and wasting prevalence were defined as the proportions of children presenting mean weight for length/height (WHZ) more than 2 standard deviations above or below 2 standard deviations from the median value of the 2006 WHO standards, respectively. Descriptive analyses include national estimates of child overweight and wasting prevalence, mean, and standard deviations of WHZ stratified by age in years. National results were pooled using the population of children aged under 5 years in each country as weight. Fractional polynomials were used to compare mean WHZ with both overweight and wasting prevalence. RESULTS: Ninety national surveys from LMICs carried out between 2010 and 2019 were included. The overall prevalence of overweight declined with age from $6.3 \%$ for infants (aged $0-11$ months) to 3.0\% in 4 years olds ( $p=0.03$ ). In all age groups, lower prevalence was observed in low-income compared to upper-middle-income countries. Wasting was also more frequent among infants, with a slight decrease between the first and second year of life, and little variation thereafter. Lower-middle-income countries showed the highest wasting prevalence in all age groups. On the other hand, mean WHZ was stable over the first 5 years of life, but the median standard deviation for WHZ decreased from 1.39 in infants to 1.09 in 4-year-old children $(p<0.001)$. For any given value of $\mathrm{WHZ}$, both overweight and wasting prevalence were higher in infants than in older children.

CONCLUSION: The higher values of WHZ standard deviations in infants suggest that declining prevalence in overweight and wasting by age may be possibly due to measurement error or rapid crossing of growth channels by infants.

International Journal of Obesity (2021) 45:2419-2424; https://doi.org/10.1038/s41366-021-00911-5

\section{BACKGROUND}

In the recent past, global health targets have shifted the focus of nutrition surveillance from undernutrition to all forms of malnutrition, which also encompasses overweight and obesity $[1,2]$. The fourth WHO Global Target recommends that countries should achieve no increase in childhood overweight by 2025 . Also, the Sustainable Development Target 2.2 is aimed at ending all forms of malnutrition (Indicator 2.2.2 on wasting and overweight) among children under 5 years of age [3]. So far, however, the Global Nutrition Report states that no country is on target to achieve these targets [4].

Overweight and obesity are considered as a global epidemic by the World Health Organization (WHO), due to their rapid increase in several countries. In 2019, it is estimated that 38.2 million $(5.6 \%)$ children under the age of 5 years were overweight or obese worldwide [5], with particularly high prevalence in uppermiddle-income and high-income countries [6]. The worldwide increase is related to the nutritional transition, including urbanization, economic growth, and globalization, leading to lifestyle changes including reduced physical activity and poor dietary habits with increased intake of highly processed, highenergy foods $[7,8]$. Whereas it is well known that prevalence of overweight increases with age after the age of 5 years [9], we were unable to find any studies showing age patterns in overweight prevalence in children aged under 5 years using data from multiple countries. Our expectation was to find an increase with age within this group.

In terms of undernutrition, 47 million children worldwide, or $6.9 \%$ of all under 5 children, suffered from wasting as of 2019 [10], and along with other forms of undernutrition accounts for 1 million child deaths annually [11]. Wasting is particularly common in South Asia, where the largest number of wasted children in the world are located [12]. Wasting, as other nutritional indicators, is closely linked to food insecurity, poor dietary quality, and disease, being concentrated in low-income settings [11, 13]. Although wasting prevalence is classically defined as being most common

\footnotetext{
${ }^{1}$ International Center for Equity in Health, Federal University of Pelotas, Pelotas, Brazil. ${ }^{2}$ Postgraduate Program in Physical Education, Federal University of Pelotas, Pelotas, Brazil. 凶email: luizaicricardo@gmail.com
} 
in the second year of life [14], recent studies show that younger children are more likely to be wasted $[12,15]$.

Even though all countries are experiencing some level of malnutrition, it is evident that differences exist regarding sociodemographic characteristics [4]. For some countries, the situation is even more concerning, with two or more extreme malnutrition scenarios simultaneously, such as high levels of undernutrition (stunting and/or wasting) and overweight, implicating in the double burden of malnutrition (DBM). This phenomenon can refer to maternal and child indicators of malnutrition in individual, household, or populational levels [16], and affects mostly low- and middle-income countries (LMICs), particularly in south and east Asia and sub-Saharan Africa [17]. A study with 93 LMICs showed that, among children under the age of 5 years, the national prevalence of DBM at individual level ranges from 0.2 to $10.9 \%$, and one-third of the countries show higher levels of DBM than would be expected by chance [18].

The Sustainable Development Goals (SDG) target 17.18 recommends disaggregation of data by income, sex, age, ethnicity, and by additional relevant characteristics in national contexts. Regarding age of the child, there is a substantial literature on inequality in stunting prevalence [19], but less attention has been given to how wasting prevalence varies with age, and even less attention to age patterns in overweight prevalence. The aim of the present study is to describe overweight and wasting prevalence among under 5 children according to age in years, in low-and middle-income countries based on data from national surveys.

\section{METHODS}

\section{Data sources and measurements}

The present analyses are based on Demographic and Health Surveys (DHS) [20] and Multiple Indicator Cluster Surveys (MICS) [21]. These nationally representative surveys collect data on reproductive, maternal, neonatal and child health and nutrition in LMICs. DHS and MICS are cross-sectional surveys which employ similar sampling methods, using multistage cluster procedures to select women of reproductive age (15-49 years) and children under 5 years of age. Both surveys use standardized questionnaires administered via face-to-face interviews by trained fieldworkers, permitting higher comparability between the surveys.

Publicly available data from DHS and MICS have been harmonized and reanalyzed by the International Center for Equity in Health (ICEH; www. equidade.org) to provide disaggregated estimates that enable the study of social and economic inequalities. The ICEH database currently comprises 412 surveys from 117 countries. The present analyses included DHS or MICS carried out from 2010 to 2019, in which anthropometric data were available for children under 5 years of age. For countries with more than one survey since 2010 , the most recent one was analyzed.

Children up to 23 months had their supine length measured, while for children aged 24-59 months the standing height was obtained. For simplicity, we refer to weight for height (WHZ) to represent both weight for length and weight for height. The measuring boards used to collect length/height include ShorrBoards (Weight and Measure, LLC, Olney, MD, USA), SECA (Hamburg, Germany), as well as locally manufactured boards in some surveys. Children under 59 months of age were weighed using portable SECA $874 \mathrm{U}$ electronic scales (Hamburg, Germany) [22, 23].

Overweight prevalence was defined as the proportion of children with mean WHZ more than 2 standard deviations above the median of the WHO reference curve $[25,26]$. Wasting prevalence was defined as the proportion of children presenting mean $\mathrm{WHZ}$ more than -2 standard deviations below the median of the WHO reference curve. Both indicators were calculated based upon the 2006 WHO Child Growth Standards $[25,26]$. Although the usual definition of $Z$ scores is based on mean, rather than median values, the growth standards use the latter to account for the asymmetric distribution of weight [27-30]. According to the WHO recommendations, we considered WHZ individual scores between -5 and +5 of the WHO Growth Standards as valid measures [24].

Standard deviations that are markedly different from the expected value of 1.0 suggest the possibility of measurement error. Using the National Center for Health Statistics (USA) reference, the WHO suggested that standard deviation values outside the range of $0.85-1.1$ for WHZ may indicate problems with anthropometric measurements [14, 24]. We assessed the variability of $\mathrm{WHZ}$ standard deviations by age and report on the number of countries with values outside 0.85-1.1.

We also analyzed overweight prevalence among children aged 2-5 years using the International Obesity Taskforce (IOTF) age- and sexadjusted BMI cut-offs for overweight and obesity in children, which correspond to the adult BMI cut-offs ( 25 and $30 \mathrm{~kg} / \mathrm{m}^{-2}$ ) [31]. The IOTF reference does not cover children aged under 2 years. Results using the IOTF BMI cut-offs for overweight are available as Supplementary Materials.

\section{Data analyses}

Descriptive analyses include estimates of child overweight and wasting at national level, stratified by age in months $(0-11,12-23,24-35,36-47$, 48-59). Ages were estimated by subtracting the birth date from the interview date. Logistic regression was used to test for within-country linear trends according to the child's age in full years, by coding each child as overweight or not overweight, and in a separate regression as wasted or not wasted.

Comparisons among the five age groups were based on Chi-square tests, providing heterogeneity $p$ values for the group differences on overweight and wasting prevalence across the five age groups. These analyses were performed separately for each outcome (overweight and wasting), analyzing all the countries included and each income group.

The graphic presentation of results included bar graphs for overweight and wasting prevalence by age and country income groups, and boxplots to show between-country variations in WHZ means and standard deviations. A scatter plot was used to relate mean $\mathrm{WHZ}$ to prevalence of overweight and wasting, using fractional polynomials to draw regression curves. All analyses were stratified by age groups and weighted by the under 5-year population in the median year of available surveys (2014). In the supplementary analyses, we used local polynomial smooth plots with confidence intervals (Ipolyci command in Stata) to show mean levels by continuous age, also with national under five populations as weights.

All country-level analyses accounted for the multistage survey design, including sampling weights and clustering. Databases were handled using Stata 16.0 (StataCorp, College Station, TX, USA) and Microsoft Excel ${ }^{\circ}$ spreadsheets (Microsoft Corp, WA, USA). Ethical clearance for the national surveys was obtained at the country level by the research institutions involved in data collection.

\section{RESULTS}

The present analyses included 90 national surveys from LMIC's carried out between 2010 and 2019 (median year=2014). Countries included in the analyses represented $87.1 \%$ of all lowincome countries in the world as of $2014,70.6 \%$ of all lower-middle and $50.9 \%$ of all upper-middle-income countries. Supplementary Table 1 shows the numbers of children in each income group for all countries included in the analyses.

The median number of children under the age of 5 years with valid anthropometric and age information across all countries was 5920 (IQR $=3263 ; 10263)$. The median numbers by age group were as follows: $0-11$ months $(N=1101 ; \mathrm{IQR}=538 ; 2100)$, $12-23$ months $(N=1137 ; \mathrm{IQR}=624 ; 2080), 24-35$ months $(N=$ $1151 ; \mathrm{IQR}=653 ; 2071), 36-47$ months $(N=1192 ; \mathrm{IQR}=612 ; 2125)$, and 48-59 months $(N=1079 ; \mathrm{IQR}=664 ; 1997)$.

Figure 1 presents overweight prevalence stratified by age and country income groups. Overall, the prevalence of overweight declined with increasing age (heterogeneity $p$ value $=0.03$ ), with a 3.3 percentage points (p.p.) of difference between the $0-11(6.3 \%)$ and the $48-59$ months (3.0\%) age groups. A similar pattern was observed when stratifying for gender (Supplementary Fig. 1) and using the IOTF classification for overweight (Supplementary Fig. 4).

Overweight prevalence was considerably lower in low-income countries in comparison with upper-middle-income countries regardless of age group (Fig. 1). In low-income countries, overweight prevalence was at least 3.8 p.p. higher in the 0-11 months group than in older age groups (heterogeneity $p$ value $<0.001)$. In lower-middle-income countries, this difference was at least 2.0 p.p., with a similar decline with age (heterogeneity $p$ value $<0.001)$. As for upper-middle-income countries, there was 
an apparent decline in prevalence with age, but it was not as pronounced as in low-income or lower-middle-income countries and did not reach statistical significance (heterogeneity $p$ value $=$ 0.082).

Supplementary Table 2 presents the national prevalence of overweight stratified by age and respective SII values. Overweight prevalence among children aged $0-11$ months varied from $1.8 \%$ in Mauritania to $23.3 \%$ in South Africa, whereas for children aged $48-59$ months age the range was from $0.1 \%$ in Madagascar to $14.5 \%$ in Egypt. Tests for linear trends in overweight prevalence showed statistically significant declines with age in 65 countries and increases with age in only three countries: El Salvador, North Macedonia, and Thailand. For the remaining 22 countries, there was no evidence of a linear trend (Supplementary Table 2). As for national estimates, Senegal was the country with the lowest national overweight prevalence among all under 5 children $(0.9 \%)$, while the highest prevalence was observed in Bosnia and Herzegovina (18.1\%) (data not shown).

Figure 2 presents wasting prevalence by age and country income groups. Wasting was more frequent among infants (0-11 months), with a slight decrease between the first and second year of life, and little variation thereafter. The same pattern was observed when stratifying for gender (Supplementary Fig. 3).

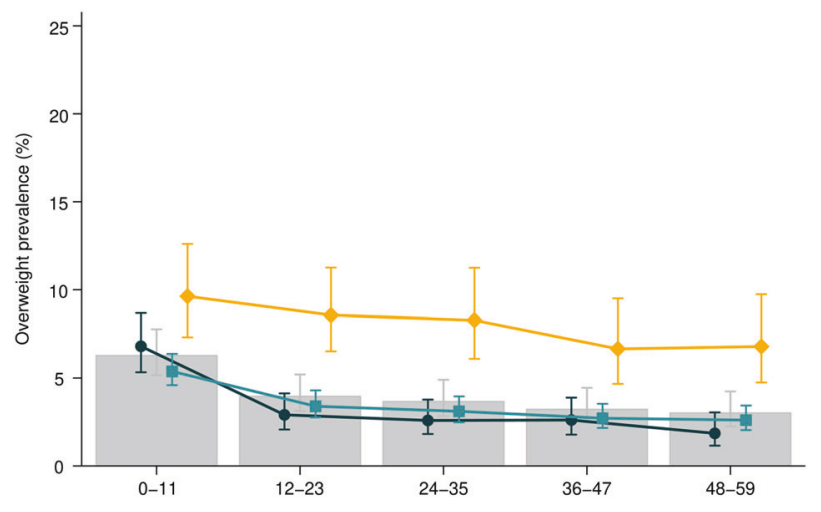

Child age (months)

Overall Low-income Lower-middle-income Upper-midle-income

Fig. 1 Overweight prevalence by age in all 90 LMIC (bars) and stratified by country income groups (symbols). Error bars represent 95\% confidence intervals for the prevalence in each group.

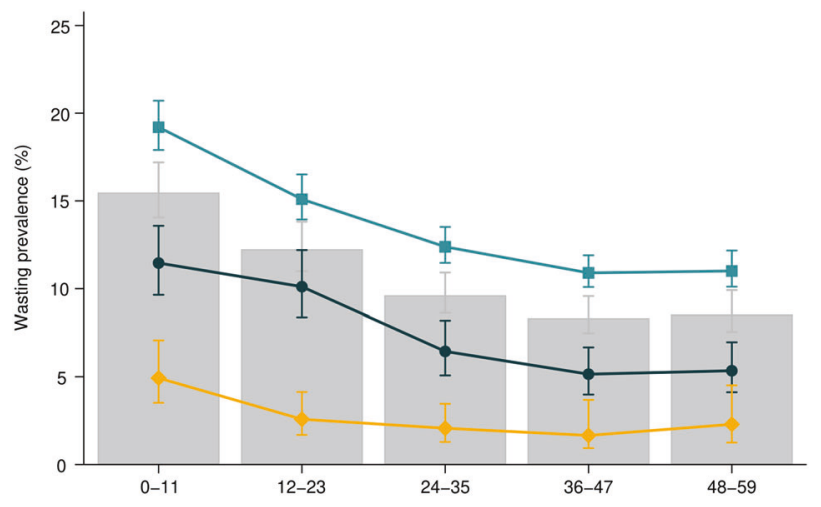

Child age (months)

Overall Low-income Lower-middle-income Upper-midle-income

Fig. 2 Wasting prevalence by age in all 90 LMIC (bars) and stratified by country income groups (symbols). Error bars represent $95 \%$ confidence intervals for the prevalence in each group.
When stratifying for country income groups, upper-middleincome countries presented the smallest prevalence, varying from $4.9 \%$ among infants to $1.7 \%$ among 3 years olds. Lower-middleincome countries showed the highest prevalence in all age groups (ranging from $19.0 \%$ among infants to $10.9 \%$ among 3 years olds). In low-income countries, prevalence ranged from $11.5 \%$ in infants to $5.2 \%$ in 3 years olds. All groups of countries showed a decline with age from infants to 3 years olds, with stability thereafter.

Wasting prevalence among children aged 0-11 months varied from $0.8 \%$ in Peru to $28.9 \%$ in India, whereas for children aged 48-59 months age the range was from $0.0 \%$ in Eswatini to $22.5 \%$ in Timor-Leste. Tests for linear trends in wasting prevalence showed statistically significant declines with age in 72 countries and increases with age only in the Maldives. For the remaining 17 countries, there was no evidence of a linear trend (Supplementary Table 3). Regarding national estimates, Peru showed the lowest national wasting prevalence $(0.5 \%)$ and Timor-Leste the highest (24.0\%) (data not shown).

As a consequence of the higher prevalence of both overweight and wasting among younger children, the proportions of children with appropriate $\mathrm{WHZ}$ (between -2 and $+2 Z$ scores) increased with age, being approximately to $78,85,88,89$, and $90 \%$ for age groups 0-11, 12-23, 24-35, 36-47, and 48-59 months, respectively (Supplementary Fig. 6).

Figure 3 presents the mean WHZ according to country income groups. Regardless of age, upper-middle-income countries showed the highest mean $\mathrm{WHZ}$, which was a little above 0 for all ages. Mean WHZ was stable in lower-middle and upper-middleincome countries, while for low-income countries a decline is observed between 0 and 12 months of age.

In Supplementary Fig. 4, the boxplots show the distribution in all countries studied of WHZ scores and respective standard deviations across age groups. Median values of the national-level $\mathrm{WHZ}$ means were close to zero, being similar among all age groups (heterogeneity $p$ value $=0.659$ ), median of the standard deviations of the national-level $\mathrm{WHZ}$ means were higher among infants than older children (heterogeneity $p$ value $<0.001$ ), for whom standard deviations were just above 1.0. Out of the 90 surveys included in the present analyses, in 86 the standard deviation for infants exceeded the maximum recommended value of 1.1 ; the corresponding numbers of surveys exceeding this cutoff were $71,49,44$, and 41 for children aged 1-4 years, respectively.

Figure 4 shows prevalence of the two outcomes (overweight and wasting) according to mean $\mathrm{WHZ}$ by age. Each dot represents one age group in a country, totaling 450 observations. The fitted curves for infants are well above the curves for older children, showing that the same level of mean WHZ is associated both with

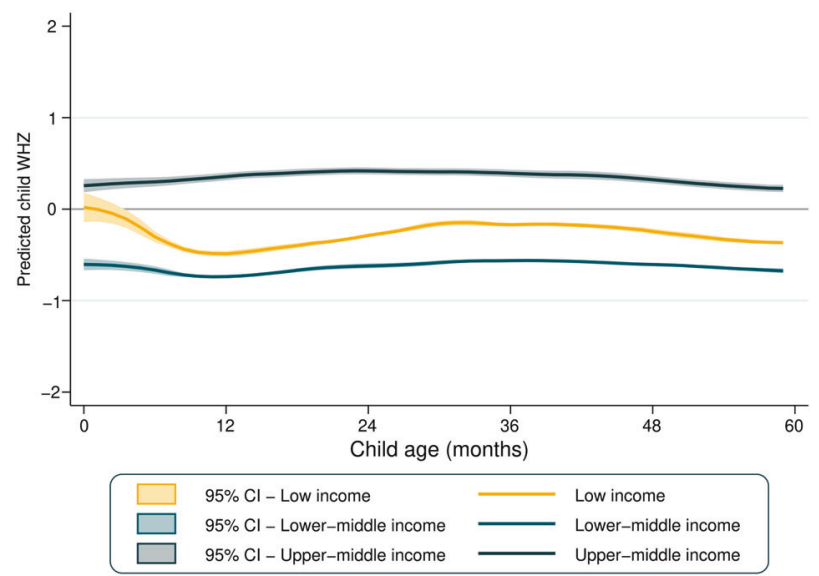

Fig. 3 The lines represent mean $\mathrm{WHZ}$ according to age in each country income group, while the shades represent $95 \%$ confidence intervals. 

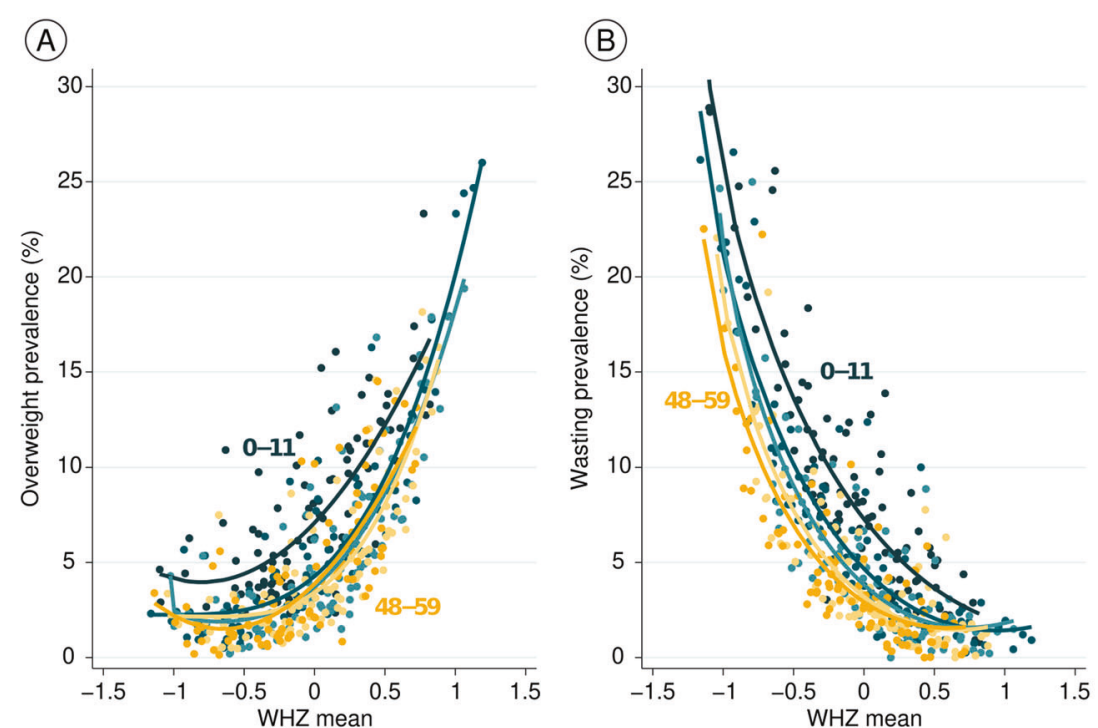

0-11 months

$12-23$ months

24-35 months

36-47 months

48-59 months

Fig. 4 Mean prevalence of overweight and wasting according to mean $\mathbf{W H Z}$, by age $(\mathbf{N}=\mathbf{4 5 0})$. Comparison between mean $\mathrm{WHZ}$ and prevalence of overweight (A) and wasting (B). Each dot represents one age group in a country, totaling 450 combinations of country and age group.

higher overweight (Fig. 4A) as well as higher wasting prevalence (Fig. 4B) in infants than for older children. This pattern is due to the wider standard deviations among infants, as shown in Supplementary Fig. 4B.

\section{DISCUSSION}

We report on patterns of child overweight and wasting prevalence in 90 LMICs, according to age in years. We are unaware of any previous publications on this issue including data from a large number of countries.

In accordance with the literature, we found that overweight prevalence showed a gradual reduction from upper-middleincome to low-income countries, with intermediate prevalence in lower-middle-income countries [32]. In contrast, wasting prevalence was higher in lower-middle-income countries than in low-income countries, an apparently paradoxical finding that is largely driven by high prevalence in the populous South Asian countries which are classified in the lower-middle-income group [12]. Wasting prevalence, as expected, was very low in uppermiddle-income countries.

Regarding age patterns, we found that prevalence of both overweight and wasting declined with age. The proportions of children classified in the normal range for $\mathrm{WHZ}$ increased from $78 \%$ in infants to $90 \%$ in 4-year-old children, and therefore the combined prevalence of wasting and overweight ranged from $22 \%$ in infants to $10 \%$ in 4 -year-old children. The latter values correspond to SDG indicator 2.2.2, which defines malnutrition as weight for height $>+2$ or $<-2$ standard deviations from the median of the WHO Child Growth Standards.

Our original hypothesis was that overweight prevalence would increase with age among under 5 children, mainly due to breastfeeding cessation and the increasing consumption of high-energy foods [33]. Yet, our results showed a decline in overweight prevalence with age in all three country income groups, and in most countries studied. This observation led us to investigate how mean $\mathrm{WHZ}$ varied with age, but no clear pattern was observed. The anthropometry literature suggests that use of mean $Z$ scores in child anthropometry has the advantage of describing the nutritional status of the entire population directly, without resorting to a subset of individuals below or above a set cutoff [14]. In addition, mean values are less likely to be affected by outliers than estimates based on the tails of the distribution.

In light of the contrasting findings of higher prevalence in young children side by side with constant mean $\mathrm{WHZ}$, we investigated how the standard deviation of WHZ measures varied with age. These analyses revealed that standard deviations were markedly higher for infants-and to a lesser extent among 1 year olds - than for older children. A possible explanation is that length or height is more difficult to measure reliably in younger children [34, 35], for whom an error of a few centimeters could markedly affect the $\mathrm{WHZ}$ value. For example, an error of only $1.5 \mathrm{~cm}$ in length or height measurement could result in $\sim 0.1$ in the $\mathrm{WHZ}$ mean and 3 percent points in wasting prevalence [36]. Earlier evaluations of DHS and MICS surveys showed heterogeneity in anthropometric data quality between and within surveys and over time, although there is evidence of improvements in more recent surveys such as those included in the present analyses [37,38]. We are unaware of any previous multicountry analyses examining how the standard deviations of WHZ vary with age in existing surveys.

To further assess the hypothesis of measurement error, we compared the mean $\mathrm{WHZ}$ with overweight and wasting prevalence in the same survey. Among infants, a given level of mean WHZ was associated with substantially higher prevalence of both overweight and wasting than among older children.

In addition to measurement quality issues, another possible explanation for the wider standard deviations observed among young children is that they are more likely to cross growth percentiles, whereas children aged 2 years or more tend to grow along the same percentile, which is known as "growth canalization." Rapid crossing of growth channels in young children could result in cross-sectional findings of high prevalence of both overweight and wasting compared to older children whose growth channels are more stable [39-41].

Our study presents some limitations. First, the percentage of countries with available surveys is not equally distributed according to World Bank income groups, as surveys were available for $87.1 \%$ of low-income, $70.6 \%$ of lower-middle-income, and $50.9 \%$ of upper- 
middle-income countries. Second, we included surveys from 2010 onward (median year $=2014$ ), and for countries without recent surveys national estimates may be outdated.

In conclusion, our study showed a pattern of decline in overweight and wasting prevalence with age in under 5 children, whereas mean $\mathrm{WHZ}$ values were constant. The decline with age is explained by wider standard deviations among young children which may be due to measurement error or by rapid crossing of growth channels. While these issues remain, it is not possible to make any firm statements about variability in prevalence of overweight by age on the basis of routinely carried out anthropometric surveys. Cohort and cross-sectional studies with high-quality anthropometry are needed to assess how overweight and wasting vary with the ages of young children.

\section{REFERENCES}

1. WHO. Decade of action on nutrition. WHO. 2019. http://www.who.int/nutrition/ decade-of-action/information_flyer/en/.

2. Nilsson M, Griggs D, Visbeck M. Policy: map the interactions between Sustainable Development Goals. Nature. 2016;534. https://pubmed.ncbi.nlm.nih.gov/ 27306173/.

3. United Nations. THE 17 GOALS. Sustainable Development. United Nations. 2015 https://sdgs.un.org/goals.

4. Global Nutrition Report. 2020 Global Nutrition Report. Global Nutrition Report. 2020 https://globalnutritionreport.org/reports/2020-global-nutrition-report/.

5. World Health Organization. Obesity and overweight. World Health Organization. 2021 https://www.who.int/news-room/fact-sheets/detail/obesity-andoverweight.

6. Di Cesare M, Sorić M, Bovet P, Miranda JJ, Bhutta Z, Stevens GA, et al. The epidemiological burden of obesity in childhood: a worldwide epidemic requiring urgent action. BMC Med. 2019;17:212. https://bmcmedicine.biomedcentral.com/ articles/10.1186/s12916-019-1449-8.

7. Kac G, Pérez-Escamilla R. Nutrition transition and obesity prevention through the life-course. Int J Obes. 2013;3:S6-8. https://www.ncbi.nlm.nih.gov/pmc/articles/ PMC4850564/.

8. Popkin BM, Adair LS, Ng SW. Global nutrition transition and the pandemic of obesity in developing countries. Nutr Rev. 2012;70:3-21. https://pubmed.ncbi. nlm.nih.gov/22221213/.

9. Rodriguez-Martinez A, Zhou B, Sophiea MK, Bentham J, Paciorek CJ, lurilli ML, et al. Height and body-mass index trajectories of school-aged children and adolescents from 1985 to 2019 in 200 countries and territories: a pooled analysis of 2181 population-based studies with 65 million participants. Lancet. 2020;396:1511-24. https://pubmed.ncbi.nlm.nih.gov/33160572/.

10. UNICEF. Malnutrition in children-UNICEF data. UNICEF. 2021. https://data.unicef. org/topic/nutrition/malnutrition/.

11. Black RE, Victora CG, Walker SP, Bhutta ZA, Christian P, De Onis M, et al. Maternal and child undernutrition and overweight in low-income and middle-income countries. Lancet. 2013;382:427-51. http://www.thelancet.com/article/ S014067361360937X/fulltext.

12. Harding KL, Aguayo VM, Webb P. Factors associated with wasting among children under five years old in South Asia: implications for action. PLoS ONE. 2018;13:e0198749. https://dx.plos.org/10.1371/journal.pone.0198749.

13. Hawkes C, Ruel MT, Salm L, Sinclair B, Branca F. Double-duty actions: seizing programme and policy opportunities to address malnutrition in all its forms. Lancet. 2020;395:142-55. http://www.thelancet.com/article/S0140673619325061/ fulltext.

14. World Health Organization (2015). Physical status: the use and interpretation of anthropometry. Report of a WHO Expert Committee. World Health Organ Tech Rep Ser. 1995;854:1-452.

15. Yalew BM. Prevalence and factors associated with stunting, underweight and wasting: a community based cross sectional study among children age 6-59 months at Lalibela Town, Northern Ethiopia. J Nutr Disorders Ther. 2014. https://doi.org/10.4172/2161-0509.1000147.

16. Kinyoki DK, Ross JM, Lazzar-Atwood A, Munro SB, Schaeffer LE, AbbasalizadFarhangi $M$, et al. Mapping local patterns of childhood overweight and wasting in low- and middle-income countries between 2000 and 2017. Nat Med. 2020;26:750-9. https://pubmed.ncbi.nlm.nih.gov/32313249/.

17. Popkin BM, Corvalan C, Grummer-Strawn LM. Dynamics of the double burden of malnutrition and the changing nutrition reality. Lancet. 2020;395:65-74. http:// www.thelancet.com/article/S0140673619324973/fulltext.

18. Lerm BR, Crochemore-Silva I, Costa JC, Victora CG. The double burden of malnutrition in under-five children at national and individual levels: observed and expected prevalence in ninety-three low- and middle-income countries. Public Health Nutr. 2020. https://pubmed.ncbi.nlm.nih.gov/32633230/.

19. Victora CG, De Onis M, Hallal PC, Blössner M, Shrimpton R. Worldwide timing of growth faltering: revisiting implications for interventions. Pediatrics. 2010;125. https://pubmed.ncbi.nlm.nih.gov/20156903/.

20. The DHS Program. Quality information to plan, monitor and improve population, health, and nutrition programs. The DHS Program. 2021. https://dhsprogram. $\mathrm{com} /$.

21. UNICEF. MICS. UNICEF. 2021. https://mics.unicef.org/.

22. ICF International. MEASURE DHS biomarker field manual. Calverton, Mariland, USA: ICF International; 2012. http://www.measuredhs.com.

23. UNICEF. MICS manual for anthropometry. UNICEF; 2017. https://www.who.int/ nutrition/publications/anthropomentry-data-quality-report-annexes.pdf? ua $=1$.

24. WHO. Recommendations for data collection, analysis and reporting on anthropometric indicators in children under 5 years old. WHO; 2019. http://www.who. int/nutrition/publications/anthropometry-data-quality-report/en/.

25. de Onis M, Branca F. Childhood stunting: a global perspective. Maternal and Child Nutrition. 2016;12:12-26. https://onlinelibrary.wiley.com/doi/full/10.1111/ men. 12231.

26. WHO. WHO child growth standards: length/height-for-age, weight-for-age, weight-for-length, weight -for-height and body mass index-for-age: methods and development. WHO; 2006. https://apps.who.int/iris/handle/10665/43413.

27. WHO Multicentre Growth Reference Study Group. WHO child growth standards: length/height-for-age, weight-for-age, weight-for-length, weight-for-height and body mass index-for-age: methods and development. WHO Multicentre Growth Reference Study Group; 2006.

28. De Onis M, Onyango AW, Borghi E, Garza C, Yang H. Comparison of the World Health Organization (WHO) Child Growth Standards and the National Center for Health Statistics/WHO international growth reference: implications for child health programmes. Public Health Nutr. 2006;9:942-7.

29. Malta DC, Porto DL, Melo FCM, Monteiro RA, Sardinha LMV, Lessa BH. Family and the protection from use of tobacco, alcohol, and drugs in adolescents, National School Health Survey. Rev Bras Epidemiol. 2011;14:166-77. http://www.scielo.br/ scielo.php?script $=$ sci_arttext\&pid $=$ S1415790X2011000500017\&lng =pt\&nrm=iso\&tlng=pt.

30. Cole TJ. The LMS method for constructing normalized growth standards. Eur J Clin Nutr. 1990;44:45-60. http://europepmc.org/article/med/2354692.

31. Cole TJ, Lobstein T. Extended international (IOTF) body mass index cut-offs for thinness, overweight and obesity. Pediatr Obes. 2012;7:284-94. https://pubmed. ncbi.nlm.nih.gov/22715120/.

32. Antonio Dassi J, Zanin A, Marcos Bagatini F, Tibola A, Barichello R. Análise da viabilidade econômico-financeira da energia solar fotovoltaica em uma Instituição de Ensino Superior do Sul do Brasil. TCPDF; 2015. www.tcpdf.org.

33. Baker P, Machado P, Santos T, Sievert K, Backholer K, Hadjikakou M, et al. Ultraprocessed foods and the nutrition transition: global, regional and national trends, food systems transformations and political economy drivers. Obes Rev. 2020;21. https://onlinelibrary.wiley.com/doi/10.1111/obr.13126.

34. Assaf $\mathrm{S}$, Kothari MT, Pullum T. DHS methodological reports 16. An assessment of the quality of DHS anthropometric data. The DHS program; 2005. https:// dhsprogram.com/publications/publication-mr16-methodological-reports.cfm.

35. Mei Z, Grummer-Strawn LM. Standard deviation of anthropometric Z-scores as a data quality assessment tool using the 2006 WHO growth standards: a cross country analysis. Bull World Health Organ. 2007;85:441-8. https://pubmed.ncbi. nlm.nih.gov/17639241/.

36. Grellety E, Golden MH. The effect of random error on diagnostic accuracy illustrated with the anthropometric diagnosis of malnutrition. PLOS ONE. 2016;11: e0168585.

37. Corsi DJ, Perkins JM, Subramanian SV. Child anthropometry data quality from demographic and health surveys, multiple indicator cluster surveys, and national nutrition surveys in the West Central Africa region: are we comparing apples and oranges? Glob Health Action. 2017;10. https://pubmed.ncbi.nlm.nih.gov/ 28641057/.

38. Perumal N, Namaste S, Qamar H, Aimone A, Bassani DG, Roth DE. Anthropometric data quality assessment in multisurvey studies of child growth. Am J Clin Nutr. 2020;112:806S-15S. https://academic.oup.com/ajcn/article/112/Supplement_2/ $806 \mathrm{~S} / 5872361$.

39. Huttly SRA, Victora CG, Barros FC, Teixeira AMB, Vaughan JP. The timing of nutritional status determination: implications for interventions and growth monitoring. Eur J Clin Nutr. 1991;45:85-95. https://jhu.pure.elsevier.com/en/ publications/the-timing-of-nutritional-status-determination-implications-for-i-4.

40. Tanner JM. Growth at adolescence. 2nd ed. Vol. 28. Oxford: Blackwell Scientific; 1962. p. 16.

41. Wilson J, Foster D, Kronenberg H, Larsen P. Williams textbook of endocrinology. 9th ed. Vol. 12. Philadelphia: Saunders; 1998. p. 113. 


\section{ACKNOWLEDGEMENTS}

This work was supported by the Bill \& Melinda Gates Foundation (grant no. INV010051/OPP1199234) and Wellcome Trust (grant no. 101815/Z/13/Z) and ABRASCO.

\section{AUTHOR CONTRIBUTIONS}

LICR, ICS, and CGV conceived this work and participated in the design, writing, and interpretation of the data; LICR performed data analysis; GGD, PARN, JSV, AJD, and CGV participated in writing and revising the paper. All authors read and approved the final manuscript.

\section{COMPETING INTERESTS}

The authors declare no competing interests.

\section{ADDITIONAL INFORMATION}

Supplementary information The online version contains supplementary material available at https://doi.org/10.1038/s41366-021-00911-5.

Correspondence and requests for materials should be addressed to L.I.C.R.
Reprints and permission information is available at http://www.nature.com/ reprints

Publisher's note Springer Nature remains neutral with regard to jurisdictional claims in published maps and institutional affiliations.

Open Access This article is licensed under a Creative Commons Attribution 4.0 International License, which permits use, sharing, adaptation, distribution and reproduction in any medium or format, as long as you give appropriate credit to the original author(s) and the source, provide a link to the Creative Commons license, and indicate if changes were made. The images or other third party material in this article are included in the article's Creative Commons license, unless indicated otherwise in a credit line to the material. If material is not included in the article's Creative Commons license and your intended use is not permitted by statutory regulation or exceeds the permitted use, you will need to obtain permission directly from the copyright holder. To view a copy of this license, visit http://creativecommons. org/licenses/by/4.0/.

(c) The Author(s) 2021 